\title{
Análise da avaliação do PSF em municípios de pequeno porte.
}

\section{Analysis of the evaluation of the FHP small cities.}

\author{
Fábio Aragão Kluthcovsky* \\ Ana Cláudia Garabeli Cavalli Kluthcovsky**
}

\begin{abstract}
Resumo
No subsistema de saúde público brasileiro o fortalecimento da Atenção Básica teve particular contribuição do Programa Saúde da Família (PSF). Atualmente, o PSF se desenvolve em cenários heterogêneos nos municípios e regiões brasileiras, espelhando a diversidade nacional e a grande variação de intensidade e formas de implantação do programa. A partir de 2005, o Ministério da Saúde (MS) propôs a institucionalização da avaliação do PSF por meio de questionários específicos. Este estudo objetivou analisar de forma crítica o instrumento de avaliação do PSF, proposto pelo MS. Tratase de um estudo descritivo-reflexivo, com base na literatura especializada. A proposta de avaliação, com desenho metodológico quantitativo, limita a apreensão dos diferentes contextos municipais. Também se faz sentir a ausência da avaliação de usuários em relação à qualidade da atenção. Outro fato importante é que a avaliação poderá ser limitada por ser de livre adesão pelos gestores municipais. Por outro lado, a proposta do MS tem o mérito de ser um processo específico e sistematizado de auto-avaliação para o PSF, com integração de diferentes atores, em um referencial metodológico tradicional em qualidade de ações de saúde.

A integração do gestor municipal ao Plano Estadual de Avaliação e Monitoramento tem o benefício de envolver estados na harmonização de desigualdades regionais, além de suprir eventuais deficiências técnicas e/ou dificuldades relativas à gestão da estratégia nos municípios. A praticidade e a "resolutividade" da avaliação do PSF proposta pelo MS certamente terão papel importante para uso do gestor e equipes no direcionamento da estratégia e tomada de decisões.
\end{abstract}

\section{Abstract}

The Family Health Program (FHP) plays an important role in the strengthening of basic care in the Brazilian public health system. Currently the FHP is spreading over the Brazilian cities and regions, being implemented with different intensity and in different forms, reflecting the diversity of the country itself. In 2005, the Ministry of Health (MH) proposed evaluating the Family Health Program by means of specific questionnaires. The aim of this descriptive reflective study is to conduct a critical analysis of the instrument proposed by the Ministry of Health for

Palavras-chave: Programa Saúde da Familia;

Avaliação em Saúde; Avaliação de Programas.
Key Words: Family Health Program;

Evaluation in Health; Evaluation in Programs.

*Médico de Família e Comunidade, Mestre em Enfermagem em Saúde Pública, Professor da UNICENTRO e Faculdade Guairacá, Brasil.

**Médica, Mestre em Enfermagem em Saúde Pública, Professora da UNICENTRO, Brasil. 
evaluating the FHP in the light of the specialized literature. The proposed quantitative evaluation methodology is limiting the comprehension of the different local contexts. Moreover, there is a lack of user-based evaluation as refers to the quality of the delivered services. Another important limitation is that the participation of the municipal administrations in the evaluation is optional. On the other hand, the proposal of the Ministry has the merit of representing a specific and systematized self-evaluation process for the FHP, integrating the opinion of different actors in a methodological approach traditionally accepted for evaluating the quality of health actions. The integration of the municipal administrations to the Evaluation and Monitoring Plan of the states has the advantage of involving the states in the harmonization of regional inequalities and in the solution of possible technical deficiencies and/ or management difficulties on municipal level. The functionality and resolutivity of the evaluation methodology proposed by the Ministry of Health will certainly play an important role in the strategic decision-making of public managers and bealth teams.

\section{Introdução}

A importância da Atenção Básica no funcionamento do sistema de saúde como um todo e o importante papel do Programa Saúde da Família (PSF) na organização da Atenção Básica em uma significativa parcela de municípios brasileiros são elementos de análise que justificam plenamente a adoção de um processo específico e sistematizado de avaliação ${ }^{1}$.

A partir de 1999, ocorreu um importante aumento no número de equipes em pequenos municípios, em função do aumento de repasse financeiro realizado pelo MS para aqueles com maiores coberturas populacionais ${ }^{2,3} . \mathrm{Na}$ seqüência, por meio de financiamentos diferenciados de custeio e investimento direcionados a municípios com mais de 100 mil habitantes, o MS buscou a expansão da estratégia em municípios de médio e grande porte ${ }^{4}$.

A expansão do PSF em diversos contextos com variados graus de implantação e diferentes níveis de cobertura gerou grande diversidade de avaliações, que buscavam quantificar resultados sob diversos pontos de vista ${ }^{5}$. Em que pese a importância das informações produzidas, ainda não estava disponível um instrumento específico de avaliação da estratégia.

Como forma de responder a esta necessidade, o Ministério da Saúde (MS) propôs a utilização de instrumentos de auto-avaliação ${ }^{1}$, com integração de respostas de diferentes atores envolvendo as equipes de gestão e operacionais, em um referencial sistêmico ${ }^{6}$, tradicionalmente aceito para avaliação de qualidade de ações de saúde. A padronização do referido instrumento busca a confiabilidade e reprodutibilidade das informações obtidas.

A iniciativa do MS em disponibilizar um instrumento de avaliação continuada é de extrema relevância, pois, com isso, as informações poderão ficar disponíveis para gestão interna, possibilitando uma melhora constante da qualidade das ações, pela importância que o PSF representa no atual contexto da saúde pública do Brasil, tanto pelo aumento crescente do número de equipes implantadas nos últimos anos, como pelos seus resultados.

Considerando a avaliação de serviços ou programas de saúde um importante e complexo tema, este estudo objetivou realizar uma análise sobre o processo de avaliação em saúde, e sobre a utilização do instrumento de autoavaliação do PSF proposto pelo MS, identificando-se suas vantagens e limitações, especialmente relacionadas às peculiaridades do PSF no contexto de pequenos municípios.

\section{O processo de avaliação}

Atualmente há consenso a respeito da necessidade de processos de avaliação nos diferentes setores de organização da atividade humana ${ }^{7}$. Por outro lado, há grande controvérsia a respeito da exata definição da avaliação ${ }^{8}$. Entende-se que uma coleta sistematizada de informações para subsidiar o estabelecimento de um juízo de valor sobre um dado objeto constitui uma avaliação formal ${ }^{9}$, com a finalidade de aplicar padrões que permitam a determinação de valor, qualidade, utilidade, efetividade ou significância ${ }^{8}$.

Apesar da importância da avaliação para a tomada de decisões, há questionamentos no meio acadêmico se uma avaliação poderia ou não ser considerada ciência. Pouvourville ${ }^{10}$ relata que a utilização de métodos científicos 
para "explorar as ações sobre a população e entender o respectivo impacto na sociedade parece para mim intimamente ligada à ciência, especialmente quando se considera que o principal propósito da última é explorar nossa realidade com métodos controlados".

Nos diferentes setores de organização das atividades humanas há diversidade no modo e finalidade dos processos de avaliação. No setor privado, a avaliação é a ferramenta para ganhos de produtividade e eficiência organizacional com satisfação do cliente. No terceiro setor, além dos benefícios anteriormente citados, a avaliação é a forma de demonstrar excelência e efetividade, no sentido de obter respaldo junto à sociedade na qual está inserido? ${ }^{7}$.

No setor público, no qual é freqüente a estruturação de ações sob a forma de programas, com o objetivo de gestão de resultados, a avaliação é condição imprescindível para demonstração de resultados, possibilitando a motivação do staff e orientando futuras intervenções. A pressão do mercado financeiro externo sobre os governos no sentido de provar a capacidade de reger o próprio crescimento e até evitar a transferência de atribuições ao setor privado, supostamente mais eficiente e efetivo, torna o embate ideológico e reforça o papel da avaliação na modernização do setor público ${ }^{10}$.

A avaliação deveria ser utilizada como cultura no setor público, como forma de fortalecimento do mesmo e racionalização de políticas em todo o mundo, tendo como finalidade o aumento da performance das intervenções ${ }^{10,11}$. Constandriopoulos ${ }^{11}$ considera a institucionalização da avaliação como uma criação de mecanismos formais de produção de informação sobre programas e políticas públicas. Ele enfatiza ainda o desafio da racionalização de recursos em um contexto de mercado globalizado com governos locais fragilizados pela necessidade de cortes em programas sociais, especialmente na área da saúde. Nesta situação, caracterizada pela tensão redistributiva ${ }^{12}$, a avaliação como processo sistemático certamente se constitui em uma importante ferramenta de gestão e tomada de decisões.

\section{A avaliação em saúde no setor público}

Apesar de todos os argumentos favoráveis à utilização da avaliação no setor público, Hartz e Pouvorville ${ }^{13}$ consideram que a mesma é prática rara no Brasil, apesar do assunto freqüentemente ocupar a agenda científica, técnica e legal no país.

Essa diferença entre retórica e prática, na realidade brasileira, pode ser atribuída a várias razões. Alguns aspectos pontuados por Travassos ${ }^{14}$, específicos para a área de saúde, incluem a falta de uma cultura política orientada por avaliação e a falta de especialistas, tanto acadêmicos como técnicos, nas áreas de avaliação de qualidade, gerência de qualidade e produção e análise de dados de saúde e documentação médica. Por outro lado, a avaliação divide a responsabilidade de tomada de decisão com segmentos mobilizados da sociedade, o que pode explicar a resistência à adoção e divulgação de resultados, em alguns casos do setor público brasileiro $^{15}$.

Novaes ${ }^{16}$ concorda com a deficiência nacional em termos de cultura e recursos humanos em avaliação, ao mesmo tempo em que identifica a oportunidade e necessidade de diversos tipos de avaliação, especificamente na área de saúde, na qual ocorreram: expansão de atenção à saúde; oferta de novas tecnologias; novos modelos assistenciais e crescimento setorial de importância política e econômica.

Tais proposições, em diferentes níveis de atenção, em geral são estruturadas sob a forma de programas, de modo que, simultaneamente à proposição, já é prevista alguma forma de avaliação a ser realizada com a implementação e o desenvolvimento do proposto.

Essa multiplicidade de programas e respectivas avaliações trazem certa dificuldade de sistematização assumida em informe técnico institucional do MS: "ainda busca-se clarear as diretrizes de uma política de avaliação, que, embora em franco processo de implantação, estabelece como desafio a superação dos obstáculos operacionais e funcionais para sua execução"17.

Hartz $^{15}$ define programa como um "Conjunto de ações visando a favorecer comportamentos adaptativos requeridos por diferentes áreas ou atividades humanas 
relacionadas com vida comunitária, escola, trabalho, saúde e bem-estar".

Um programa em geral pressupõe a necessidade de articulação de ações de diferentes atores com responsabilidades diversas, em geral demandando práticas intersetoriais. O processo de avaliação é complexo pela perspectiva integradora das diferentes contribuições ${ }^{10}$. Esta necessidade de integração decorre da natureza complexa do processo de produção saúde-doença, em que interagem diferentes sistemas e processos, de modo que o resultado na atenção à saúde não depende exclusivamente de um determinado serviço de saúde ${ }^{14}$.

Nesta complexidade, a avaliação de programas públicos se constitui em um desafio metodológico, no qual se busca identificar a relação interativa de negociação entre parcerias, e iterativa, na qual se manifesta, ou não, a relação entre ação e resultados. O grau de implantação dos programas em diferentes contextos é outra importante questão para não se incorrer no erro das avaliações precoces ${ }^{10}$.

Para dar conta do desafio metodológico, Hartz ${ }^{18}$ defende o modelo de avaliação orientado pela teoria explicativa do respectivo funcionamento. Neste modelo, denominado theory-driven evaluation, prima-se por uma construção lógica, com base em pesquisas prévias, teorias de ciências sociais e experiência de gestores e avaliadores, nas quais se buscam respostas às questões centrais do programa: "Qual o problema ou comportamento visado?”, “Como a população se caracteriza?”, “Quais as condições do contexto?", "Quais os ingredientes ativos no programa?” (Questão relativa ao conteúdo do programa necessário para produzir os efeitos desejados).

Este modelo teórico diz respeito à lógica de causa e efeito do programa no sentido plural, a partir da análise dos subprogramas, em uma articulada e hierárquica relação do tipo se - então, que compõe o todo ${ }^{19}$.

Em relação à metodologia utilizada na avaliação de programas de saúde, pode-se perceber a construção de um progressivo consenso em torno da utilização combinada de técnicas de análises qualitativas e quantitativas ${ }^{15,16,18,19,20} \mathrm{em}$ um enfoque feito sob medida, ou sur mesure para o programa avaliado, como prefere Hartz ${ }^{15}$. Esta construção metodológica é diferente dos desenhos experimentais ou quase experimentais tradicionalmente utilizados, sendo freqüente a utilização de estudo de caso para avaliação de programas na área da saúde s5,18,19,21,22. $^{2}$.

Independentemente do desenho metodológico, em todos os tipos de avaliação está presente a idéia de qualidade. Embora a palavra qualidade seja amplamente utilizada, sua definição não é tarefa simples, e, não obstante, não pode ficar em termos abstratos. Isto em função de que, para o avaliador, a qualidade deve se tornar um objeto mensurável.

Em termos gerais, a qualidade é verificada quando da atribuição de juízo ao objeto avaliado resulta um valor positivo. Estabelecer o juízo de valor positivo implica na utilização de critérios implícitos e explícitos ${ }^{23}$.

Avedis Donabedian é o autor consensualmente utilizado como referencial teórico na avaliação de qualidade

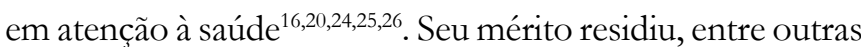
razões, na capacidade de sistematizar a apropriação de qualidade em termos de serviços, ações e programas de saúde. Desta forma, asseverou que qualidade deve representar um julgamento positivo em relação à técnica envolvida na atenção à saúde e no relacionamento interpessoal entre cliente e prestador ${ }^{23}$.

Nesta perspectiva, o autor reconheceu a existência de várias definições de qualidade em serviços de saúde ${ }^{6}$ e adotou uma forma mais ampliada de entendê-la, incluindo múltiplos aspectos, tais como: consideração dos potenciais benefício e dano que uma dada intervenção pode determinar na saúde do paciente, informação completa ao paciente ou representante legítimo das considerações anteriores, nãoutilização de práticas de pouca ou nenhuma utilidade, responsabilidade de fazer o melhor pelo paciente em dadas circunstâncias e consideração dos custos envolvidos na atenção ao paciente.

Outro aspecto do enfoque de qualidade em saúde é a consideração do papel do consumidor, no caso os usuários dos serviços de saúde, nos ganhos de qualidade através de: retroalimentações fornecidas diretamente aos prestadores, 
ou, indiretamente, por via administrativa, mecanismos de mercado e ação política. Qualidade é "a medida em que são utilizadas as circunstâncias mais favoráveis para o alcance de melhoria na saúde"27.

A riqueza deste referencial para a qualidade das ações de saúde pode ser verificada na capacidade de unificar enfoques diferenciados em um conjunto de critérios complementares denominados de atributos da atenção em saúde. No clássico artigo denominado Os sete pilares da qualidade $^{27}$, os critérios de definição de qualidade em atenção à saúde foram divididos em eficácia, efetividade, eficiência, otimização, aceitabilidade, legitimidade e eqüidade.

Em outros trabalhos ${ }^{6,28}$, retomou-se um modelo de avaliação derivado da abordagem de análise de sistemas, no qual é adotado um paradigma com distinção de etapas denominadas de estrutura, processo e resultado ou impacto. A abordagem de análise de sistemas traz vantagens em função da formulação científica, da orientação para gestão, da objetividade quantitativa e replicabilidade, da ênfase em relações de causa-efeito entre os componentes do sistema e sua lógica conceitual ${ }^{29}$.

Este marco conceitual é largamente utilizado na avaliação de programas de saúde, sendo inclusive a fundamentação teórica utilizada pelo MS para avaliação de programas específicos ${ }^{21,24}$. Outros autores acrescentaram ao modelo original outros elementos, conservando o núcleo central de integração e a interdependência dos itens analisa$\operatorname{dos}^{30}$. Apesar da divisão em etapas, o modelo proposto permite flexibilidade, sendo usual a dificuldade em diferenciá-las ${ }^{31}$.

O MS tem empreendido esforços no sentido de disponibilizar aos gestores municipais instrumentos de gerência e avaliação no nível local, com um importante recorte na atenção básica, de forma especial em relação ao $\mathrm{PSF}^{1}$. A ênfase no PSF pode ser justificada em função da sua significativa expansão nos últimos anos e pela importância como porta de entrada do sistema de saúde como um todo.

\section{A avaliação do PSF}

O PSF tem como objetivo a reorganização da prática assistencial em substituição ao modelo tradicional de assistência à saúde. A assistência está centrada na família a partir de seu ambiente físico e social, em território definido, possibilitando às equipes de Saúde da Família (ESF), de caráter multiprofissional, melhor compreensão do processo saúdedoença e da necessidade de intervenções que vão além das práticas curativas. Apresenta-se como uma estratégia que prioriza ações de promoção, proteção e recuperação da saúde dos indivíduos, além de reafirmar e incorporar os princípios básicos do Sistema Único de Saúde (SUS), a universalização, descentralização, integralidade e participação da comunidade ${ }^{32}$.

No ano de 2006, havia um total de 26.729 ESF implantadas em 5.106 municípios brasileiros, representando $91,8 \%$ destes, cobrindo 46,2\% da população brasileira, o que corresponde a cerca de 85,7 milhões de pessoas ${ }^{33}$.

A adesão dos municípios à estratégia Saúde da Família foi variável conforme o porte dos municípios. Assim, os municípios menores implantaram a estratégia de modo mais precoce e com maior facilidade do que os municípios de grande porte ${ }^{1}$. A partir de novembro de 1999, com o incremento no valor dos incentivos repassados pelo MS para os municípios em direta proporção com as faixas de cobertura populacional pelo programa ${ }^{34}$, a lógica financeira passou a ser o melhor argumento junto à administração de pequenos municípios para a adoção do PSF. Neste contexto, um pequeno número de equipes representa 100\% de cobertura do PSF, com alcance do valor máximo de repasse financeiro por equipe de saúde.

Já os grandes municípios brasileiros apresentam certas características, como complexidade sociossanitária, existência de modelos de atenção em saúde estabelecidos, organização urbana e perfil e formação dos profissionais ${ }^{1}$, além de concentrarem mais da metade da população do país ${ }^{4}$.

Em termos de Brasil, a cobertura populacional do Saúde da Família aumentou 600\% no período de 1998 a 2004. Contudo, quanto menor o município, mais alta a cobertura do PSF e mais acelerada sua expansão. Em 2004, 65,29\% da população dos municípios com menos de 20 mil habitantes era atendida pelo programa, e apenas $27,50 \%$ da população 
dos municípios com 80 mil ou mais habitantes estava coberta pelo programa ${ }^{35}$.

A fim de superar as limitações quanto à expansão do PSF em grandes centros, foi desenvolvido pelo MS o Projeto de Expansão e Consolidação do Saúde da Familia (PROESF), com apoio do Banco Mundial - BIRD -, estruturado em três componentes de avaliação: incentivar e ampliar o número de equipes dirigido aos municípios com mais de 100 mil habitantes; formar profissionais para o trabalho na estratégia, independente do porte, e fortalecer os processos de monitoramento e avaliação, independente do porte ${ }^{4}$.

Tornar a avaliação como ação sistemática no sistema público de saúde é assumida pelo MS como passo fundamental para dar qualidade às ações desenvolvidas para o cumprimento dos princípios basilares do SUS. Por essa razão, um dos objetivos do PROESF foi criar uma cultura e uma sistemática de avaliação e monitoramento em saúde, beneficiando municípios que adotaram o $\mathrm{PSF}^{3}$.

Antes do PROESF, o Pacto da Atenção Básica, feito anualmente, apresentava alguns indicadores relativos à estratégia do $\mathrm{PSF}^{36}$. A partir de 2005, no entanto, a Coordenação de Avaliação da Atenção Básica passou a propor um processo de avaliação específico para o PSF, intitulado Avaliação para Melhoria da Qualidade da Estratégia Saúde da Família ${ }^{1}$. Neste processo de avaliação, qualidade em saúde é definida como "o grau de atendimento a padrões de qualidade estabelecidos frente às normas e aos protocolos que organizam as ações e práticas, assim como aos conhecimentos técnicos e científicos atuais, respeitando valores culturalmente aceitos" 29,37 .

Utilizando referenciais misto de Donabedian ${ }^{29}$ e Uchimura e Bosi ${ }^{37}$, o MS assume a necessidade de apreender o impacto da atuação das ESF, nos respectivos contextos.

Esta proposta é de difícil operacionalização, pois pressupõe um processo de avaliação extremamente complexo, sugerindo a adoção de uma linha de estudo de caso para diferentes contextos ${ }^{15,30}$.

As diretrizes da avaliação proposta pelo MS caracterizam-se como um processo auto-avaliativo, livre adesão pelos gestores municipais, ausência de incentivos ou punições financeiras ou outras relacionadas aos resultados, utilização de aplicativo digital para alimentação de banco de dados e emissão de relatórios via internet e integração às atividades desenvolvidas no âmbito dos Planos Estaduais de Monitoramento e Avaliação da Atenção Básica ${ }^{1}$.

A proposta adota a utilização de cinco instrumentos de auto-avaliação, com ênfase nos processos de trabalho. Pela inerente possibilidade de pronta intervenção quando da identificação dos problemas, em uma linha de avaliação para gestão. Aspectos de estrutura e resultado são tomados como parâmetros para avaliação da qualidade, a partir de uma visão dinâmica de estágios de qualidade inter-relacionados, porém com menor ênfase em relação àqueles indicadores de processo. Vale ressaltar que, na avaliação no nível local, são focados dois componentes nucleares ou unidades de análise para a avaliação: a gestão municipal do Saúde da Família e as equipes $^{1}$.

A gestão municipal da estratégia Saúde da Família é avaliada prioritariamente pelos instrumentos 1 e 2 preenchidos, respectivamente, pelo gestor municipal e coordenação da estratégia. Responsáveis pelas unidades de Saúde da Família, integrantes das ESF e profissionais de nível superior respondem os instrumentos 3 , 4 e 5, respectivamente, gerando as principais informações para avaliação das ESF em relação a 1 :

- Infra-estrutura: insumos, equipes, materiais, recursos humanos e ambiente físico; existência e utilização de manuais de procedimentos, guias de conduta, sistemas informatizados, dentre outros

- Processos relativos a: aspectos organizativos (planejamento e programação, abrangência das ações e participação comunitária), técnico-científicos (competência técnico-científica e protocolização do atendimento) e relação interpessoal (acolhimento e comunicação interpessoal).

- Resultados: diretos (decorrem da ampliação do acesso, adequação e efetividade das ações desenvolvidas) e de saúde da população (relacionados a indicadores epidemiológicos em termos de internações por doenças evitáveis, morbidade e mortalidade e dependem de uma grande proporção de fatores relacionados à prestação do cuidado nos demais 
níveis de atenção do sistema, bem como do envolvimento e participação de outros setores e atores da área social).

Em que pese a integração dos diferentes níveis de governo no processo de avaliação proposto, o nível local será responsável pela concretização, ou não, da referida proposta e pela viabilização, ou não, das ações de melhoria da qualidade.

O nível local, após análise dos instrumentos, será qualificado em cinco padrões crescentes de qualidade, variando entre: padrão E (Qualidade Elementar), padrão D (Qualidade em Desenvolvimento), padrão C (Qualidade Consolidada), padrão B (Qualidade Boa) e padrão A (Qualidade Avançada). Esses estágios devem refletir momentos de um processo de evolução da qualidade nos serviços ${ }^{1}$.

Segundo o MS, os principais beneficiários dessa iniciativa seriam os próprios usuários do sistema de saúde, já que a melhora contínua na qualidade do Saúde da Família representará melhoria no acesso, maior resolubilidade e humanização dos serviços ${ }^{1}$. Contudo, se faz sentir a ausência da avaliação de usuários em relação à qualidade da atenção, considerados por Santos, Uchimura e Lang ${ }^{38}$ a razão da existência dos serviços de saúde.

Além disso, o desenho metodológico quantitativo limita a apreensão dos diferentes contextos municipais, já que os municípios com população igual ou inferior a $20 \mathrm{mil}$ habitantes apresentam características próprias, de modo que a avaliação não deveria obedecer ao mesmo padrão que municípios de diferentes portes ${ }^{10}$. Como exemplos dessas características próprias dos pequenos municípios, podem ser citados, dentre outros, a dificuldade de fixação de profissionais de saúde integrantes das equipes; dificuldade de entendimento, por parte do corpo técnico gestor da estratégia, da avaliação como instrumento para gestão; unidades do PSF cobrindo grandes áreas rurais com baixa densidade populacional e dificuldades particulares nas condições de trabalho; dependência dos municípios pólo para atenção especializada, dificultando a referência e contra-referência.

Essas limitações poderiam ser identificadas por estudos específicos, com utilização de técnicas qualitativas e quantitativas aplicadas em paralelo ao instrumento sistematizado, naquelas situações singulares, podendo ainda esclarecer elementos facilitadores e dificultadores da estratégia.

Outra questão importante é que a avaliação proposta pelo MS poderá ser limitada por uma baixa adesão de municípios, já que o processo de avaliação depende da livre adesão do gestor municipal, o que poderia ser minimizado pela adoção de incentivos para aqueles que apresentassem evidências da utilização das informações para gestão interna e melhoria da qualidade das ações.

Por outro lado, a proposta do MS tem o mérito de ser um processo específico e sistematizado de auto-avaliação para o PSF, com integração de diferentes atores, em um referencial metodológico tradicional em qualidade de ações de saúde.

A integração do gestor municipal ao Plano Estadual de Avaliação e Monitoramento tem o potencial de desencadear e incrementar a participação dos estados na proposição e implementação de ações com vistas à harmonização de desigualdades regionais nos diferentes níveis de atenção, em especial na Atenção Básica. A coordenação estadual também pode suprir eventuais deficiências técnicas e/ou dificuldades relativas à gestão da estratégia nos municípios. Contudo, o adequado desempenho do gestor estadual se constitui em nó crítico neste processo, especialmente para aqueles municípios com limitado corpo técnico e/ou dificuldades relativas à gestão da estratégia.

\section{Considerações finais}

A importância da Atenção Básica na organização do sistema de saúde como um todo e o incontestável papel do PSF na organização da Atenção Básica em uma significativa parcela de municípios brasileiros são elementos de análise que justificam plenamente a adoção de um processo específico e sistematizado de avaliação.

O desenho metodológico da proposta do MS, com técnica quantitativa, não prevê a identificação dos diferentes contextos em que a estratégia está atuando, bem como a não-avaliação das expectativas dos usuários em relação à qualidade da atenção.

Por outro lado, a utilização de instrumentos de autoavaliação, com integração de respostas de diferentes atores, 
envolvendo as equipes de gestão e operacionais em um referencial metodológico tradicionalmente aceito para avaliação de qualidade de ações de saúde é uma garantia no sentido da confiabilidade e reprodutibilidade das informações obtidas. São características que sugerem que a proposta do MS deverá ser mantida ao longo do tempo.

Apesar das limitações, é extremamente oportuna a iniciativa do MS quanto à proposta de avaliação continuada com disponibilização de informações para gestão interna, possibilitando uma melhora constante da qualidade das ações. Por outro lado, na medida em que o sistema produzisse as informações, seria interessante que tanto gestor municipal quanto equipes pudessem acessar facilmente os resultados da avaliação e eventuais propostas de correção, e tomada de decisões e direcionamento das ações fossem acompanhadas com respaldo pelos demais níveis de governo.

\section{Referências}

1.Brasil. Ministério da Saúde. Avaliação para Melhoria da Qualidade da Estratégia Saúde da Família. Documento Técnico. Brasilia (DF): Ministério da Saúde; 2005. 110p.

2.Kluthcovsky FA. Avaliação do processo de expansão do Programa Saúde da Família em um município do Sul do Brasil [Dissertação]. Ribeirão Preto, Escola de Enfermagem de Ribeirão Preto, Universidade de São Paulo; 2005.

3.World Bank. Human Development Sector Management Unit. Brazil Country Management Unit Latin America and the Caribbean Region. Report. N 233353 BR. The Brazil Family Health Extension Program. 2002. Disponível em: http:// www-wds.worldbank.org/servlet/WDSContentServer/ WDSP/IB/2002/03/15/000094946_02022804005161/ Rendered/PDF/multiopage.pdf. Acesso em: 30 ago. 2007. 4.Leal PRM. Grandes cidades, grandes desafios. Revista Brasileira de Saúde da Família. 2002; 6:44-48.

5.Lentsck MH. Avaliação do Programa Saúde da Família: uma revisão. Trabalho de Conclusão de Curso de Graduação em Enfermagem. Guarapuava, Universidade Estadual do CentroOeste; 2007.

6.Donabedian A. Explorations in Quality Assessment and
Monitoring. The Definition of Quality and Approaches to Its Assessment. Ann Arbor, MI: Health Administration Press; 1980. $163 \mathrm{p}$.

7.O'Connel B 1993. Preface. In: Gray ST. Leadership IS: a vision of evaluation Washington: Independent Sector; 1993. p. iii.

8.Worthen BR, Sanders JR, Fitzpatrick JL. Introduction to evaluation. In:___. Program evaluation: alternative approaches and practical guidelines. New York: Longman Publishers USA; 1997.558 p.

9.Sanders JR. Uses of evaluation as a means toward organizational effectiveness. In: Gray ST. Leadership IS: a vision of evaluation. Washington: Independent Sector; 1993. p. 1318.

10.Pouvorville G. Evaluation the French chefs are still searching for "la nouvelle cuisine". Cadernos de Saúde Pública. 1999; 15(2):248-250.

11.Constandriopoulos AP. Is the institutionalization of evaluation sufficient to guarantee its practice? Cadernos de Saúde Pública. 1999; 15(2):253-256.

12.Campos FCC. Gestão intergovernamental e financiamento do Sistema Único de Saúde: apontamentos para os gestores municipais. In: Brasil. Ministério da Saúde. Gestão municipal de saúde: textos básicos. 20. ed. Brasília (DF): Ministério da Saúde; 2001. p. 79-109.

13.Hartz ZMA, Pouvorville G. A avaliação da eficiência em saúde: a eficiência em questão. Ciência \& Saúde Coletiva. 1998; III(1):68-82.

14.Travassos C. Debate on the paper by Zulmira M. A. Hartz. Cadernos de Saúde Pública. 1999; 15(2): 246-247.

15.Hartz ZMA. Avaliação dos programas de saúde: perspectivas teórico metodológicas e políticas institucionais. Ciência \& Saúde Coletiva. 1999; 4(2):341-353.

16. Novaes HMD. Avaliação de programas, serviços e tecnologias em saúde. Revista de Saúde Pública. 2000; 34(5):547-549.

17.Felisberto E. Monitoramento e avaliação na atenção básica: novos horizontes. Revista Brasileira de Saúde Materno-infantil. 2004; 4(3):317-321.

18.Hartz ZMA. Avaliação em saúde: dos modelos conceituais 
à prática na análise da implantação de programas. Rio de Janeiro: Fiocruz; 1997. 131 p.

19.Hartz ZMA. Institutionalizing the evaluation of helth programs and policies in France: cuisine internationale over fast food and sur mesure over ready-made. Cadernos de Saúde Pública. 1999; 15(2):229-260.

20.Perrin E. Some thoughts on outcomes research, quality improvement, and performance measurement. Medical Care. 2002; 40(6 suppl):III89-91.

21.Brasil. Ministério da Saúde. Avaliação da Implantação do Programa de Saúde da Família em dez grandes centros urbanos. Síntese dos principais resultados. Brasília (DF): Ministério da Saúde; 2002. 228p.

22.Yin RK. Estudo de caso: planejamento e métodos. Trad. Daniel Grassi. 2. ed. Porto Alegre: Bookman; 2001. 205 p. 23.Donabedian A. Reflections on the effectiveness of quality assurance. In: Palmer RH, Donabedian A, Povar G. Striving for quality in health care: an inquiry into policy and practice. Michigan: Health Administration Press; 1991. p. 61-128.

24. Brasil. Ministério da Saúde. Sistema de avaliação metodologia de avaliação dos subprojetos do componente I do projeto REFORSUS. 1. ed. Brasília (DF): Ministério da Saúde; 1999. p. 11-17.

25.Palmer RH. Process-based measures of quality: the need for detailed clinical data in large health care databases. Annal of Internal Medicine. 1997; 127(8 suppl):733-738.

26.Trad LAB, Bastos ACS. O impacto sócio-cultural do Programa Saúde da Família (PSF): uma proposta de avaliação. Cadernos de Saúde Pública. 1998; 14(2):429-435.

27.Donabedian A. The seven pillars of quality. Archives of Pathology and Laboratory Medicine. 1990; 114:1115 -1118. 28.Donabedian A. The role of outcomes in quality assessment and assurance. Quality review bulletin. 1992; 18:356-360.

29.Donabedian A. Twenty years of research on the quality of medical care 1964 -1984. Evaluation and the Health Professions; 1985. 8(3):243-265.

30.Handler A, Issel M, Turnock B. A conceptual framework to measure performance of the public health system. American Journal of Public Health. 2001; 91(8):1235-1239. 31.Pereira MG. Qualidade dos serviços de saúde. In:
Epidemiologia: teoria e prática. 1. ed. Rio de Janeiro: Guanabara Koogan; 1995; p. 538-560.

32.Brasil. Ministério da Saúde. Programa Saúde da Família. Brasília (DF): Ministério da Saúde; 2001. 36p.

33.Brasil. Ministério da Saúde. Departamento de Atenção Básica. Atenção Básica e a Saúde da Família. Disponível em: http://dtr2004.saude.gov.br/dab/abnumeros.php. Acesso em: 25 nov. 2007.

34.Brasil. Ministério da Saúde. Portaria n 1329/GM de 12 de novembro de 1999. Estabelece nova sistemática para o cálculo do incentivo financeiro ao Programa Saúde da Família, parte integrante do Piso da Atenção Básica - PAB, 1999. Disponível em:http://www.saude.gov.br/portaria/ portaria.htm. Acesso em: 22 out. 2007.

35.Brasil. Ministério da Saúde. Informe da Atenção Básica. Pesquisa nacional registra a evolução do Saúde da Família e sua relação com indicadores de saúde. Brasília(DF): Ministério da Saúde; 2005, ano VII, nov.-dez. 2p.

36.Brasil. Ministério da Saúde. Portaria no 21 de 5 de janeiro de 2005. Aprova a Relação de Indicadores da Atenção Básica - 2005. Portal da Saúde. Brasília (DF): Ministério da Saúde; 2005. 66p. Disponível em: http://tabnet.datasus.gov.br/cgi/ siab/pacto2005/ portaria21.pdf. Acesso em: 01 mar. 2007. 37.Uchimura KY, Bosi MLM. Qualidade e subjetividade na avaliação de programas e serviços em saúde. Cadernos de Saúde Pública. 2002; 18(6):1561-1569.

38.Santos SM, Uchimura KY, Lang, RMF. Percepção dos usuários do Programa Saúde da Família: uma experiência local. Cadernos de Saúde Coletiva. 2005; 13(3): 687-704.

\section{Endereço para correspondência:}

R. Simeão Varela de Sá, n. 3

Guarapuava - PR

CEP: 85.040-080

\section{Endereço eletrônico:}

anafabio@brturbo.com.br 